\title{
Some New Integral Inequalities for $n$-Times Differentiable Trigonometrically Convex Functions
}

\author{
Kerim Bekar $^{1}$ \\ ${ }^{1}$ Department of Mathematics, Faculty of Sciences and Arts, Giresun University-Giresun-Turkey
}

\section{Article Info}

Keywords: Convex function, trigonometrically convex function, Hölder Integral inequality, Power-Mean Integral inequality and Hölder-Isscan integral inequality.

2010 AMS: 26A51, 26D10, $26 D 15$.

Received: 28 November 2019

Accepted: 14 September 2020

Available online: 29 September 2020

\begin{abstract}
In this manuscript, by using an integral identity together with both the Hölder, Hölder-İscan and the Power-mean integral inequalities we obtain several new inequalities for $n$-time differentiable trigonometrically convex functions.
\end{abstract}

\section{Preliminaries}

$\Omega: I \subseteq \mathbb{R} \rightarrow \mathbb{R}$ be a convex function on the interval $I$ of real numbers and $r, s \in I$ with $r<s$. The inequality

$$
\Omega\left(\frac{r+s}{2}\right) \leq \frac{1}{s-r} \int_{r}^{s} \Omega(u) d u \leq \frac{\Omega(r)+\Omega(s)}{2}
$$

is well known in the literature as Hermite-Hadamard's $(\mathrm{H}-\mathrm{H})$ integral inequality for convex functions [13]. The classical $\mathrm{H}-\mathrm{H}$ inequality provides estimates of the mean value of a continuous convex or concave function. In recent years, significant improvements and generalizations have been found on convexity theory and $\mathrm{H}-\mathrm{H}$ inequality; see for example [1-6, 8, 13].

Definition 1.1. A function $\Omega: I \subseteq \mathbb{R} \rightarrow \mathbb{R}$ is said to be convex if the inequality

$$
\Omega(\varepsilon r+(1-\varepsilon) s) \leq \varepsilon \Omega(r)+(1-\varepsilon) \Omega(s)
$$

is valid for all $r, s \in I$ and $\varepsilon \in[0,1]$. If this inequality reverses, then $\Omega$ is said to be concave on interval $I \neq \varnothing$.

For some inequalities, generalizations and applications concerning convexity see $[2-4,6,11-15]$. Recently, in the literature there are so many papers about $n$-times differentiable functions on several kinds of convexities. In references [2,4,8,14], readers can find some results about this issue. Many papers have been written by a number of mathematicians concerning inequalities for different classes of convex functions see for instance the recent papers $[1,3,5,6]$ and the references within these papers.

In [9], Kadakal gave the concept of the trigonometrically convex functions and related Hermite-Hadamard type inequalities.

Definition 1.2 ([9]). A non-negative function $\Omega: I \rightarrow \mathbb{R}$ is called trigonometrically convex function on interval $[r, s]$, if for each $r, s \in I$ and $\varepsilon \in[0,1]$

$$
\Omega(\varepsilon r+(1-\varepsilon) s) \leq\left(\sin \frac{\pi \varepsilon}{2}\right) \Omega(r)+\left(\cos \frac{\pi \varepsilon}{2}\right) \Omega(s) .
$$

If this inequality reveresed, then the function is called trigonometrically concave. 
Theorem 1.3 ( [9]). Let the function $\Omega:[r, s] \rightarrow \mathbb{R}, s>0$, be a trigonometrically convex function. If $0 \leq r<s$ and $\Omega \in L[r, s]$, then the following inequality holds:

$$
\frac{1}{s-r} \int_{r}^{s} \Omega(x) d x \leq \frac{2}{\pi}[\Omega(r)+\Omega(s)] .
$$

Remark 1.4. It is easily seen that, if the function $\Omega:[r, s] \rightarrow \mathbb{R}, s>0$, be a trigonometrically concave function, then for $0 \leq r<s$ and $\Omega \in L[r, s]$, then the following inequality holds:

$$
\frac{1}{s-r} \int_{r}^{s} \Omega(x) d x \geq \frac{2}{\pi}[\Omega(r)+\Omega(s)] .
$$

Theorem 1.5 ( [9]). Let the function $\Omega:[r, s] \rightarrow \mathbb{R}, s>0$, be a trigonometrically convex function. If $0 \leq r<s$ and $\Omega \in L[r, s]$, then the following inequalities holds:

$$
\Omega\left(\frac{s+r}{2}\right) \leq \frac{\sqrt{2}}{s-r} \int_{r}^{s} \Omega(x) d x
$$

Remark 1.6. It is easily seen that, if the function $\Omega:[r, s] \rightarrow \mathbb{R}, s>0$, be a trigonometrically concave function, then for $0 \leq r<s$ and $\Omega \in L[r, s]$, then the following inequality holds:

$$
\Omega\left(\frac{a+b}{2}\right) \geq \frac{\sqrt{2}}{s-r} \int_{r}^{s} \Omega(x) d x
$$

A refinement of Hölder integral inequality better approach than Hölder integral inequality can be given as follows:

Theorem 1.7 (Hölder-İşcan Integral Inequality [7]). Let $p>1$ and $\frac{1}{p}+\frac{1}{q}=1$. If $f$ and $g$ are real functions defined on interval $[r, s]$ and if $|f|^{p},|g|^{q}$ are integrable functions on $[r, s]$ then

$$
\int_{r}^{s}|f(x) g(x)| d x \leq \frac{1}{s-r}\left\{\left(\int_{r}^{s}(s-x)|f(x)|^{p} d x\right)^{\frac{1}{p}}\left(\int_{r}^{s}(s-x)|g(x)|^{q} d x\right)^{\frac{1}{q}}+\left(\int_{r}^{s}(x-r)|f(x)|^{p} d x\right)^{\frac{1}{p}}\left(\int_{r}^{s}(x-r)|g(x)|^{q} d x\right)^{\frac{1}{q}}\right\}
$$

Let $0<r<s$, throughout this paper we will use

$$
\begin{aligned}
A(r, s) & =\frac{r+s}{2} \\
L_{p}(r, s) & =\left(\frac{s^{p+1}-r^{p+1}}{(p+1)(s-r)}\right)^{\frac{1}{p}}, r \neq s, p \in \mathbb{R}, p \neq-1,0
\end{aligned}
$$

for the arithmetic and generalized logarithmic mean, respectively.

\section{Main Results}

We will use the following Lemma for obtain our main results.

Lemma 2.1 ( [10]). Let $\Omega: I \subseteq \mathbb{R} \rightarrow \mathbb{R}$ be n-times differentiable mapping on $I^{\circ}$ for $n \in \mathbb{N}$ and $\Omega^{(n)} \in L[r, s]$, where $r, s \in I^{\circ}$ with $r<s$, we have the identity

$$
\sum_{k=0}^{n-1}(-1)^{k}\left(\frac{\Omega^{(k)}(s) s^{k+1}-\Omega^{(k)}(r) r^{k+1}}{(k+1) !}\right)-\int_{r}^{s} \Omega(x) d x=\frac{(-1)^{n+1}}{n !} \int_{r}^{s} x^{n} \Omega^{(n)}(x) d x
$$

where an empty sum is understood to be nil.

Theorem 2.2. For $n \in \mathbb{N}$; let $\Omega: I \subseteq(0, \infty) \rightarrow \mathbb{R}$ be $n$-times differentiable function on $I^{\circ}$ and $r, s \in I^{\circ}$ with $r<s$. If $\Omega^{(n)} \in L[r, s]$ and $\left|\Omega^{(n)}\right|^{q}$ for $q>1$ is trigonometrically convex function on the interval $[r, s]$, then the following inequality holds:

$$
\left|\sum_{k=0}^{n-1}(-1)^{k}\left(\frac{\Omega^{(k)}(s) s^{k+1}-\Omega^{(k)}(r) r^{k+1}}{(k+1) !}\right)-\int_{r}^{s} \Omega(x) d x\right| \leq \frac{s-r}{n !}\left(\frac{4}{\pi}\right)^{\frac{1}{q}} L_{n p}^{n}(s, r) A^{\frac{1}{q}}\left(\left|\Omega^{(n)}(r)\right|^{q},\left|\Omega^{(n)}(s)\right|^{q}\right) .
$$

Proof. If the function $\left|\Omega^{(n)}\right|^{q}$ for $q>1$ is trigonometrically convex on the interval $[r, s]$, using Lemma 2.1, the Hölder integral inequality and

$$
\left|\Omega^{(n)}(x)\right|^{q}=\left|\Omega^{(n)}\left(\frac{s-x}{s-r} r+\frac{x-r}{s-r} s\right)\right|^{q} \leq \sin \frac{\pi(s-x)}{2(s-r)}\left|\Omega^{(n)}(r)\right|^{q}+\cos \frac{\pi(s-x)}{2(s-r)}\left|\Omega^{(n)}(s)\right|^{q},
$$


we get

$$
\begin{aligned}
& \left|\sum_{k=0}^{n-1}(-1)^{k}\left(\frac{\Omega^{(k)}(s) s^{k+1}-\Omega^{(k)}(r) r^{k+1}}{(k+1) !}\right)-\int_{r}^{s} \Omega(x) d x\right| \\
& \leq \frac{1}{n !} \int_{r}^{s} x^{n}\left|\Omega^{(n)}(x)\right| d x \\
& \leq \frac{1}{n !}\left(\int_{r}^{s} x^{n p} d x\right)^{\frac{1}{p}}\left(\int_{r}^{s}\left|\Omega^{(n)}(x)\right|^{q} d x\right)^{\frac{1}{q}} \\
& \leq \frac{1}{n !}\left(\int_{r}^{s} x^{n p} d x\right)^{\frac{1}{p}}\left(\int_{r}^{s}\left[\sin \frac{\pi(s-x)}{2(s-r)}\left|\Omega^{(n)}(a)\right|^{q}+\cos \frac{\pi(s-x)}{2(s-r)}\left|\Omega^{(n)}(b)\right|^{q}\right] d x\right)^{\frac{1}{q}} \\
& =\frac{1}{n !}\left(\int_{r}^{s} x^{n p} d x\right)^{\frac{1}{p}}\left(\left|\Omega^{(n)}(r)\right|^{q} \int_{r}^{s} \sin \frac{\pi(s-x)}{2(s-r)} d x+\left|\Omega^{(n)}(s)\right|^{q} \int_{r}^{s} \cos \frac{\pi(s-x)}{2(s-r)} d x\right)^{\frac{1}{q}} \\
& =\frac{1}{n !}\left(\frac{s^{n p+1}-r^{n p+1}}{n p+1}\right)^{\frac{1}{p}}\left(\frac{2}{\pi}(s-r)\left|\Omega^{(n)}(r)\right|^{q}+\frac{2}{\pi}(s-r)\left|\Omega^{(n)}(s)\right|^{q}\right)^{\frac{1}{q}} \\
& =\frac{1}{n !}(s-r)^{\frac{1}{p}}(s-r)^{\frac{1}{q}}\left(\frac{4}{\pi}\right)^{\frac{1}{q}}\left(\frac{s^{n p+1}-r^{n p+1}}{(n p+1)(s-r)}\right)^{\frac{1}{p}}\left[\frac{\left|\Omega^{(n)}(r)\right|^{q}+\left|\Omega^{(n)}(s)\right|^{q}}{2}\right]^{\frac{1}{q}} \\
& =\frac{s-r}{n !}\left(\frac{4}{\pi}\right)^{\frac{1}{q}}\left[\frac{s^{n p+1}-r^{n p+1}}{(n p+1)(s-r)}\right]^{\frac{1}{p}}\left[\frac{\left|\Omega^{(n)}(r)\right|^{q}+\left|\Omega^{(n)}(s)\right|^{q}}{2}\right]^{\frac{1}{q}} \\
& =\frac{s-r}{n !}\left(\frac{4}{\pi}\right)^{\frac{1}{q}} L_{n p}^{n}(r, s) A^{\frac{1}{q}}\left(\left|\Omega^{(n)}(r)\right|^{q},\left|\Omega^{(n)}(s)\right|^{q}\right) .
\end{aligned}
$$

Corollary 2.3. Under the conditions Theorem 2.2 for $n=1$ we have the following inequality:

$$
\left|\frac{\Omega(s) s-\Omega(s) s}{s-r}-\frac{1}{s-r} \int_{r}^{s} \Omega(x) d x\right| \leq\left(\frac{4}{\pi}\right)^{\frac{1}{q}} L_{p}(r, s)\left[\frac{\left|\Omega^{\prime}(r)\right|^{q}+\left|\Omega^{\prime}(s)\right|^{q}}{2}\right]^{\frac{1}{q}} .
$$

Proposition 2.4. Let $r, s \in(0, \infty)$ with $r<s, q>1$ and $m \in(-\infty, 0] \cup[1, \infty) \backslash\{-2 q,-q\}$, we have

$$
L_{\frac{m}{q}+1}^{\frac{m}{q}+1}(r, s) \leq\left(\frac{4}{\pi}\right)^{\frac{1}{q}} L_{p}(r, s) A^{\frac{1}{q}}\left(r^{m}, s^{m}\right)
$$

Proof. Under the assumption of the Proposition, let $\Omega(x)=\frac{q}{m+q} x^{\frac{m}{q}+1}, x \in(0, \infty)$. Then

$$
\left|\Omega^{\prime}(x)\right|^{q}=x^{m}
$$

is trigonometrically convex on $(0, \infty)$ and the result follows directly from Corollary 2.3 .

Theorem 2.5. For $n \in \mathbb{N}$; let $\Omega: I \subseteq(0, \infty) \rightarrow \mathbb{R}$ be $n$-times differentiable function on $I^{\circ}$ and $r, s \in I^{\circ}$ with $r<s$. If $\Omega^{(n)} \in L[r, s]$ and $\left|\Omega^{(n)}\right|^{q}$ for $q>1$ is trigonometrically convex function on the interval $[r, s]$, then the following inequality holds:

$$
\begin{aligned}
& \left|\sum_{k=0}^{n-1}(-1)^{k}\left(\frac{\Omega^{(k)}(s) s^{k+1}-\Omega^{(k)}(r) r^{k+1}}{(k+1) !}\right)-\int_{r}^{s} \Omega(x) d x\right| \\
& \leq \frac{(s-r)^{\frac{1}{q}}}{n !}\left(\left[s L_{n p}^{n p}(r, s)-L_{n p+1}^{n p+1}(r, s)\right]\right)^{\frac{1}{p}}\left(\frac{4}{\pi^{2}}\left|\Omega^{(n)}(r)\right|^{q}+\frac{2(\pi-2)}{\pi^{2}}\left|\Omega^{(n)}(s)\right|^{q}\right)^{\frac{1}{q}} \\
& +\frac{(s-r)^{\frac{1}{q}}}{n !}\left(\left[L_{n p+1}^{n p+1}(r, s)-a L_{n p}^{n p}(r, s)\right]\right)^{\frac{1}{p}}\left(\frac{2(\pi-2)}{\pi^{2}}\left|\Omega^{(n)}(r)\right|^{q}+\frac{4}{\pi^{2}}\left|\Omega^{(n)}(s)\right|^{q}\right)^{\frac{1}{q}} .
\end{aligned}
$$

Proof. If the function $\left|\Omega^{(n)}\right|^{q}$ for $q>1$ is trigonometrically convex on the interval $[r, s]$, using Lemma 2.1, the Hölder-İşcan integral inequality and

$$
\left|\Omega^{(n)}(x)\right|^{q}=\left|\Omega^{(n)}\left(\frac{s-x}{s-r} r+\frac{x-r}{s-r} s\right)\right|^{q} \leq \sin \frac{\pi(s-x)}{2(s-r)}\left|\Omega^{(n)}(r)\right|^{q}+\cos \frac{\pi(s-x)}{2(s-r)}\left|\Omega^{(n)}(s)\right|^{q},
$$


112

Universal Journal of Mathematics and Applications

we get

$$
\begin{aligned}
& \left|\sum_{k=0}^{n-1}(-1)^{k}\left(\frac{\Omega^{(k)}(s) s^{k+1}-\Omega^{(k)}(r) r^{k+1}}{(k+1) !}\right)-\int_{r}^{s} \Omega(x) d x\right| \\
& \leq \frac{1}{n !} \int_{r}^{s} x^{n}\left|\Omega^{(n)}(x)\right| d x \\
& \leq \frac{1}{n !}\left(\int_{r}^{s} x^{n p} d x\right)^{\frac{1}{p}}\left(\int_{r}^{s}\left|\Omega^{(n)}(x)\right|^{q} d x\right)^{\frac{1}{q}} \\
& \leq \frac{1}{n !(s-r)}\left(\int_{r}^{s}(s-x) x^{n p} d x\right)^{\frac{1}{p}}\left(\int_{r}^{s}(s-x)\left[\sin \frac{\pi(s-x)}{2(s-r)}\left|\Omega^{(n)}(a)\right|^{q}+\cos \frac{\pi(s-x)}{2(s-r)}\left|\Omega^{(n)}(b)\right|^{q}\right] d x\right)^{\frac{1}{q}} \\
& +\frac{1}{n !(s-r)}\left(\int_{r}^{s}(x-r) x^{n p} d x\right)^{\frac{1}{p}}\left(\int_{r}^{s}(x-r)\left[\sin \frac{\pi(s-x)}{2(s-r)}\left|\Omega^{(n)}(r)\right|^{q}+\cos \frac{\pi(s-x)}{2(s-r)}\left|\Omega^{(n)}(s)\right|^{q}\right] d x\right)^{\frac{1}{q}} \\
& =\frac{1}{n !(s-r)}\left(\int_{r}^{s}(s-x) x^{n p} d x\right)^{\frac{1}{p}}\left(\left|\Omega^{(n)}(r)\right|^{q} \int_{r}^{s}(s-x) \sin \frac{\pi(s-x)}{2(s-r)} d x+\left|\Omega^{(n)}(s)\right|^{q} \int_{r}^{s}(b-x) \cos \frac{\pi(s-x)}{2(s-r)} d x\right)^{\frac{1}{q}} \\
& +\frac{1}{n !(s-r)}\left(\int_{r}^{s}(x-r) x^{n p} d x\right)^{\frac{1}{p}}\left(\left|\Omega^{(n)}(r)\right|^{q} \int_{r}^{s}(x-r) \sin \frac{\pi(s-x)}{2(s-r)} d x+\left|\Omega^{(n)}(s)\right|^{q} \int_{r}^{s}(x-r) \cos \frac{\pi(s-x)}{2(s-r)} d x\right)^{\frac{1}{q}} \\
& =\frac{1}{n !(s-r)}\left((s-r)\left[s L_{n p}^{n p}(r, s)-L_{n p+1}^{n p+1}(r, s)\right]\right)^{\frac{1}{p}}\left(\frac{4(s-r)^{2}}{\pi^{2}}\left|\Omega^{(n)}(r)\right|^{q}+\frac{2(\pi-2)(s-r)^{2}}{\pi^{2}}\left|\Omega^{(n)}(s)\right|^{q}\right)^{\frac{1}{q}} \\
& +\frac{1}{n !(s-r)}\left((s-r)\left[L_{n p+1}^{n p+1}(r, s)-r L_{n p}^{n p}(r, s)\right]\right)^{\frac{1}{p}}\left(\frac{2(\pi-2)(s-r)^{2}}{\pi^{2}}\left|\Omega^{(n)}(r)\right|^{q}+\frac{4(s-r)^{2}}{\pi^{2}}\left|\Omega^{(n)}(s)\right|^{q}\right)^{\frac{1}{q}} \\
& =\frac{(s-r)^{\frac{1}{q}}}{n !}\left(\left[s L_{n p}^{n p}(r, s)-L_{n p+1}^{n p+1}(r, s)\right]\right)^{\frac{1}{p}}\left(\frac{4}{\pi^{2}}\left|\Omega^{(n)}(r)\right|^{q}+\frac{2(\pi-2)}{\pi^{2}}\left|\Omega^{(n)}(s)\right|^{q}\right)^{\frac{1}{q}} \\
& +\frac{(s-r)^{\frac{1}{q}}}{n !}\left(\left[L_{n p+1}^{n p+1}(r, s)-r L_{n p}^{n p}(r, s)\right]\right)^{\frac{1}{p}}\left(\frac{2(\pi-2)}{\pi^{2}}\left|\Omega^{(n)}(r)\right|^{q}+\frac{4}{\pi^{2}}\left|\Omega^{(n)}(s)\right|^{q}\right)^{\frac{1}{q}} .
\end{aligned}
$$

Theorem 2.6. For $n \in \mathbb{N}$; let $\Omega: I \subseteq(0, \infty) \rightarrow \mathbb{R}$ be $n$-times differentiable function on $I^{\circ}$ and $r, s \in I^{\circ}$ with $r<s$. If $\Omega^{(n)} \in L[r, s]$ and $\left|\Omega^{(n)}\right|^{q}$ for $q \geq 1$ is trigonometrically convex on the interval $[r, s]$, then the following inequality holds:

$$
\left|\sum_{k=0}^{n-1}(-1)^{k}\left(\frac{\Omega^{(k)}(s) s^{k+1}-\Omega^{(k)}(r) r^{k+1}}{(k+1) !}\right)-\int_{r}^{s} \Omega(x) d x\right| \leq \frac{1}{n !}(s-r)^{1-\frac{1}{q}} L_{n}^{n\left(1-\frac{1}{q}\right)}\left\{\left|\Omega^{(n)}(r)\right|^{q} S_{1}(r, s)+\left|\Omega^{(n)}(s)\right|^{q} S_{2}(r, s)\right\}^{\frac{1}{q}},
$$

where

$$
S_{1}(r, s)=\int_{r}^{s} x^{n} \sin \frac{\pi(s-x)}{2(s-r)} d x, \quad S_{2}(r, s)=\int_{r}^{s} x^{n} \cos \frac{\pi(s-x)}{2(s-r)} d x .
$$

Proof. From Lemma 2.1 and Power-mean integral inequality, we have

$$
\begin{aligned}
& \left|\sum_{k=0}^{n-1}(-1)^{k}\left(\frac{\Omega^{(k)}(s) s^{k+1}-\Omega^{(k)}(r) r^{k+1}}{(k+1) !}\right)-\int_{r}^{s} \Omega(x) d x\right| \\
& \leq \frac{1}{n !} \int_{r}^{s} x^{n}\left|\Omega^{(n)}(x)\right| d x \\
& \leq \frac{1}{n !}\left(\int_{r}^{s} x^{n} d x\right)^{1-\frac{1}{q}}\left(\int_{r}^{s} x^{n}\left|\Omega^{(n)}(x)\right|^{q} d x\right)^{\frac{1}{q}} \\
& \leq \frac{1}{n !}\left(\int_{r}^{s} x^{n} d x\right)^{1-\frac{1}{q}}\left(\int_{r}^{s} x^{n}\left[\sin \frac{\pi(s-x)}{2(s-r))}\left|\Omega^{(n)}(r)\right|^{q}+\cos \frac{\pi(s-x)}{2(s-r))}\left|\Omega^{(n)}(s)\right|^{q}\right] d x\right)^{\frac{1}{q}} \\
& =\frac{1}{n !}\left(\int_{r}^{s} x^{n} d x\right)^{1-\frac{1}{q}}\left(\left|\Omega^{(n)}(r)\right|^{q} \int_{r}^{s} x^{n} \sin \frac{\pi(s-x)}{2(s-r))} d x+\left|\Omega^{(n)}(s)\right|^{q} \int_{r}^{s} x^{n} \cos \frac{\pi(s-x)}{2(s-r))^{2}} d x\right)^{\frac{1}{q}} \\
& \left.=\frac{1}{n !}(s-r)\right)^{1-\frac{1}{q}}\left[\frac{s^{n+1}-r^{n+1}}{(n+1)(s-r)}\right]^{1-\frac{1}{q}}\left\{\left|\Omega^{(n)}(s)\right|^{q} S_{1}(r, s)+\left|\Omega^{(n)}(r)\right|^{q} S_{2}(r, s)\right\}^{\frac{1}{q}} \\
& =\frac{1}{n !}(s-r)^{1-\frac{1}{q}} L_{n}^{n\left(1-\frac{1}{q}\right)}\left\{\left|\Omega^{(n)}(r)\right|^{q} S_{1}(r, s)+\left|\Omega^{(n)}(s)\right|^{q} S_{2}(r, s)\right\}^{\frac{1}{q}} .
\end{aligned}
$$


Corollary 2.7. Under the conditions Theorem 2.6 for $n=1$ we have the following inequality:

$$
\left|\frac{\Omega(s) s-\Omega(s) s}{s-r}-\frac{1}{s-r} \int_{r}^{s} \Omega(x) d x\right| \leq\left(\frac{r+s}{2}\right)^{1-\frac{1}{q}}\left[\frac{2 \pi s-4(s-r)}{\pi^{2}}\left|\Omega^{\prime}(r)\right|^{q}+\frac{4(s-r)-2 \pi r}{\pi^{2}}\left|\Omega^{\prime}(s)\right|^{q}\right]^{\frac{1}{q}} .
$$

Proposition 2.8. Let $r, s \in(0, \infty)$ with $r<s, q>1$ and $m \in(-\infty, 0] \cup[1, \infty) \backslash\{-2 q,-q\}$, we have

$$
L_{\frac{m}{q}+1}^{\frac{m}{q}+1}(r, s) \leq A^{1-\frac{1}{q}}(r, s)\left[\frac{2 \pi s-4(s-r)}{\pi^{2}} r^{m}+\frac{4(s-r)-2 \pi r}{\pi^{2}} s^{m}\right]^{\frac{1}{q}} .
$$

Proof. The result follows directly from Corollary 2.7 for the function

$$
\Omega(x)=\frac{q}{m+q} x^{\frac{m}{q}+1}, x \in(0, \infty) .
$$

This completes the proof of Proposition.

Corollary 2.9. Using Proposition 2.8. for $m=1$, we have following inequality:

$$
L_{\frac{1}{q}+1}^{\frac{1}{q}+1}(r, s) \leq A^{1-\frac{1}{q}}(r, s)\left[\frac{4(s-r)^{2}}{\pi^{2}}\right]^{\frac{1}{q}} .
$$

Corollary 2.10. Using Proposition 2.8 for $q=1$, we have following inequality:

$$
L_{m+1}^{m+1}(r, s) \leq \frac{2 \pi s-4(s-r)}{\pi^{2}} r^{m}+\frac{4(s-r)-2 \pi r}{\pi^{2}} s^{m} .
$$

Corollary 2.11. Using Corollary 2.10 for $m=1$, we have following inequality:

$$
L_{2}^{2}(r, s) \leq \frac{4(s-r)^{2}}{\pi^{2}} .
$$

Corollary 2.12. With the conditions of the Theorem 2.6 for $q=1$ we have the following inequality:

$$
\left|\sum_{k=0}^{n-1}(-1)^{k}\left(\frac{\Omega^{(k)}(s) s^{k+1}-\Omega^{(k)}(r) r^{k+1}}{(k+1) !}\right)-\int_{r}^{s} \Omega(x) d x\right| \leq \frac{1}{n !}\left\{\left|\Omega^{(n)}(r)\right| S_{1}(r, s)+\left|\Omega^{(n)}(s)\right| S_{2}(r, s)\right\}
$$

Theorem 2.13. For $n \in \mathbb{N}$; let $\Omega: I \subset(0, \infty) \rightarrow \mathbb{R}$ be n-times differentiable function on $I^{\circ}$ and $r, s \in I^{\circ}$ with $r<s$. If $\Omega^{(n)} \in L[r, s]$ and $\left|\Omega^{(n)}\right|^{q}$ for $q>1$ is trigonometrically concave on the interval $[a, b]$, then the following inequality holds:

$$
\left|\sum_{k=0}^{n-1}(-1)^{k}\left(\frac{\Omega^{(k)}(s) s^{k+1}-\Omega^{(k)}(r) r^{k+1}}{(k+1) !}\right)-\int_{r}^{s} \Omega(x) d x\right| \leq \frac{s-r}{n !}\left(\frac{1}{2}\right)^{\frac{1}{2 q}} L_{n p}^{n}(r, s)\left|\Omega^{(n)}\left(\frac{r+s}{2}\right)\right| .
$$

Proof. Since $\left|\Omega^{(n)}\right|^{q}$ for $q>1$ is trigonometrically concave on the interval $[r, s]$, with respect to Hermite-Hadamard inequality we can write

$$
\int_{r}^{s}\left|\Omega^{(n)}(x)\right|^{q} d x \leq \frac{s-r}{\sqrt{2}}\left|\Omega^{(n)}\left(\frac{r+s}{2}\right)\right|^{q} .
$$

Using Lemma 2.1 and the Hölder integral inequality we have

$$
\begin{aligned}
\left|\sum_{k=0}^{n-1}(-1)^{k}\left(\frac{\Omega^{(k)}(s) s^{k+1}-\Omega^{(k)}(r) r^{k+1}}{(k+1) !}\right)-\int_{r}^{s} \Omega(x) d x\right| & \leq \frac{1}{n !} \int_{r}^{s} x^{n}\left|\Omega^{(n)}(x)\right| d x \\
& \leq \frac{1}{n !}\left(\int_{r}^{s} x^{n p} d x\right)^{\frac{1}{p}}\left(\int_{r}^{s}\left|\Omega^{(n)}(x)\right|^{q} d x\right)^{\frac{1}{q}} \\
& \leq \frac{1}{n !}\left(\int_{r}^{s} x^{n p} d x\right)^{\frac{1}{p}}\left(\frac{s-r}{\sqrt{2}}\left|f^{(n)}\left(\frac{r+s}{2}\right)\right|^{q}\right)^{\frac{1}{q}} \\
& =\frac{s-r}{n !}\left(\frac{1}{2}\right)^{\frac{1}{2 q}}\left[\frac{s^{n p+1}-r^{n p+1}}{(n p+1)(s-r)}\right]^{\frac{1}{p}}\left|\Omega^{(n)}\left(\frac{r+s}{2}\right)\right| \\
& =\frac{s-r}{n !}\left(\frac{1}{2}\right)^{\frac{1}{2 q}} L_{n p}^{n}(r, s)\left|\Omega^{(n)}\left(\frac{r+s}{2}\right)\right| .
\end{aligned}
$$

Corollary 2.14. With the conditions of the Theorem 2.13 for $n=1$ we have the following inequality:

$$
\left|\frac{\Omega(s) s-\Omega(s) s}{s-r}-\frac{1}{s-r} \int_{r}^{s} \Omega(x) d x\right| \leq\left(\frac{1}{2}\right)^{\frac{1}{2 q}} L_{p}(r, s)\left|\Omega^{\prime}\left(\frac{r+s}{2}\right)\right| .
$$




\section{References}

[1] M. Alomari, M. Darus and S.S. Dragomir, New inequalities of Hermite-Hadamard type for functions whose second derivatives absolute values are quasi-convex, Tamkang J. Math, 41 (4), 2010, 353-359.

[2] P. Cerone, S.S. Dragomir and J. Roumeliotis, Some Ostrowski type inequalities for n-time differentiable mappings and applications, Demonstratio Math., 32 (4) (1999), 697-712.

[3] S.S. Dragomir and C.E.M. Pearce, Selected Topics on Hermite-Hadamard Inequalities and Applications, RGMIA Monographs, Victoria University, 2000

[4] D.Y. Hwang, Some Inequalities for n-time Differentiable Mappings and Applications, Kyung. Math. Jour., 43 (2003), $335-343$.

[5] İ. İşcan, S. Turhan, S. Maden, Some Hermite-Hadamard-Fejer type inequalities for Harmonically convex functions via Fractional Integral, New Trends Math. Sci., 4 (2) (2016), 1-10.

[6] İ. İşcan, M. Kunt, Hermite-Hadamard-Fejer type inequalities for quasi-geometrically convex functions via fractional integrals, J. Math., Volume 2016, Article ID 6523041, 7 pages.

[7] İ., İşcan, New refinements for integral and sum forms of Hölder inequality, J. Inequal. Appl., (2019) 2019:304, 11 pages.

[8] W.D. Jiang, D.W. Niu, , Y. Hua and F. Qi, Generalizations of Hermite-Hadamard inequality to n-time differentiable function which are s-convex in the second sense, Analysis (Munich), 32 (2012), 209-220.

[9] H. Kadakal, Hermite-Hadamard type inequalities for trigonometrically convex functions, Sci. Stud. Res. Ser. Math. Info., 28(2), (2018), 19-28.

[10] S. Maden, H. Kadakal, M. Kadakal and İ. İşcan, Some new integral inequalities for n-times differentiable convex and concave functions. J. Non. Sci. Appl., 10 12,(2017), 6141-6148.

[11] S. Özcan, Some Integral Inequalities for Harmonically $(\alpha, s)$-Convex Functions, J. Funct. Spaces, 2019, Article ID 2394021, 8 pages (2019).

[12] S. Özcan, and İ. İşcan, Some new Hermite-Hadamard type inequalities for s-convex functions and their applications, J. Inequal. Appl., Article number: 2019:201 (2019).

[13] J.E. Pečarić, F. Porschan and Y.L. Tong, Convex Functions, Partial Orderings, and Statistical Applications, Academic Press Inc., 1992.

[14] S.H. Wang, B.Y. Xi and F. Qi, Some new inequalities of Hermite-Hadamard type for n-time differentiable functions which are m-convex, Analysis (Munich), 32 (2012), 247-262.

[15] B.Y. Xi and F. Qi, Some integral inequalities of Hermite-Hadamard type for convex functions with applications to means, J. Funct. Spaces Appl., 2012, http://dx.doi.org/10.1155/2012/980438. 